Artículo Original

\title{
Construyendo y entendiendo la ocupación en contextos privados de libertad: experiencias de familias con hijos en situación de discapacidad en campamentos de refugiados
}

Construindo e compreendendo a ocupação em contextos privados de liberdade: experiências de famílias com crianças em situação de deficiência em campos de refugiados

\section{Building and understanding occupation in contexts deprived of freedom: experiences of families with children in a situation of disability in refugee camps}

Pablo Cantero-Garlito ${ }^{a}$ (D), Marta Rodríguez-Hernández ${ }^{a}$ (D), Carmen Cipriano-Crespo ${ }^{\text {(D) }}$ ${ }^{a}$ University of Castilla-La Mancha - UCLM, Talavera de la Reina, Spain.

Cómo citar: Cantero-Garlito, P., Rodríguez-Hernández, M., \& Cipriano-Crespo, C. (2021).

Construyendo y entendiendo la ocupación en contextos privados de libertad: experiencias de familias con hijos en situación de discapacidad en campamentos de refugiados. Cadernos Brasileiros de Terapia Ocupacional, 29, e2843. https://doi.org/10.1590/2526-8910.ctoAO2121

\begin{abstract}
Resumen
Introducción: Desde la Terapia Ocupacional se ha prestado una especial atención a la descripción y el análisis de las ocupaciones que tienen lugar en contextos de mayor vulnerabilidad y precariedad social en los que el desempeño y la participación ocupacional en condiciones de salud, bienestar y sentido personal pueden verse comprometidos de forma significativa. Objetivo: Describir y explicar la ocupación desde un enfoque cultural en familias con hijos en situación de discapacidad que residen en un contexto privado de libertad como son los campamentos de refugiados saharauis. Método: Estudio descriptivo transversal, de naturaleza etnográfica, realizado en los campamentos de refugiados saharauis de Tindouf (Argelia). Resultados: En los campamentos, el tiempo invertido en el desarrollo de las actividades de la vida diaria dista mucho de occidente, partiendo de que el propio concepto 'tiempo' presenta un significado muy distinto. Los quehaceres diarios de los saharauis están marcados por tres "tiempos": (1) la luz del sol, (2) el rezo y (3) la hora del té, siendo las dos últimas las ocupaciones más significativas de la población refugiada. El significado de la cultura parecía ser una
\end{abstract}


parte integral de la práctica profesional, así como las circunstancias vitales con las que convivían las familias con miembros en situación de discapacidad. Conclusión: La Terapia Ocupacional debe verse interpelada a ofrecer oportunidades de vida cotidiana más digna para todas las personas, apoyando y protegiendo los derechos de participación ocupacional de todos los sujetos, especialmente de aquellos que experimentan situaciones de mayor vulnerabilidad.

Palabras clave: Terapia Ocupacional, Personas con Discapacidad, Cultura, Refugiados, África Sub-Sahara.

\section{$\underline{\text { Abstract }}$}

Introduction: From Occupational Therapy, special attention has been paid to the description and analysis of the occupations that take place in contexts of greater vulnerability and social precariousness in which occupational performance and participation in conditions of health, well-being, and personal sense can be significantly compromised. Objective: To describe and explain the occupation from a cultural approach in families with children with disabilities living in a context deprived of freedom such as the Sahrawi refugee camps. Method: Crosssectional descriptive study, of an ethnographic nature, carried out in the Saharan refugee camps of Tindouf (Algeria). Results: In the camps, the time invested in the development of daily life activities is far from the West, since the very concept of 'time' has a very different meaning. The daily chores of the Saharawis are marked by three "times": (1) sunlight, (2) prayer, and (3) tea time, the last two being the most significant occupations of the refugee population. The meaning of culture seemed to be an integral part of professional practice, as well as the life circumstances with which families with disabled members lived. Conclusion: Occupational Therapy must be challenged to offer opportunities for a more dignified daily life for all people, supporting and protecting the rights of occupational participation of all subjects, especially those who experience situations of greater vulnerability.

Keywords: Occupational Therapy, Disabled Persons, Culture, Refugees, SubSaharan Africa.

\section{$\underline{\text { Resumo }}$}

Introduçáa: Do ponto de vista da Terapia Ocupacional, foi dada atenção especial à descrição e análise das ocupaçôes que ocorrem em contextos de maior vulnerabilidade e precariedade social nos quais o desempenho ocupacional e a participação em condiçôes de saúde, bem-estar e senso pessoal podem ser significativamente comprometidos. Objetivo: Descrever e explicar a ocupação a partir de uma abordagem cultural em famílias com crianças deficientes que vivem em um contexto privado de liberdade, como os campos de refugiados saharauis. Método: Estudo descritivo transversal, de natureza etnográfica, realizado nos campos de refugiados saarianos de Tindouf (Argélia). Resultados: Nos campos, o tempo gasto no desenvolvimento das atividades da vida diária está longe do Ocidente, uma vez que o próprio conceito de "tempo" tem um significado muito diferente. As tarefas diárias dos saharauis são marcadas por três "tempos": (1) luz solar, (2) oração, e (3) hora do chá, sendo as duas últimas as ocupaçốes mais significativas da população refugiada. $\mathrm{O}$ significado da cultura parecia ser parte 
integrante da prática profissional, assim como as circunstâncias de vida com as quais as famílias com membros deficientes viviam. Conclusáo: A terapia ocupacional deve ser desafiada a oferecer oportunidades para uma vida diária mais digna para todas as pessoas, apoiando e protegendo os direitos de participação ocupacional de todos os sujeitos, especialmente aqueles que vivenciam situaçóes de maior vulnerabilidade.

Palavras-chave: Terapia Ocupacional, Pessoas com Deficiência, Cultura, Refugiado, África Subsaariana.

\section{Introducción}

Aunque la preocupación de los y las terapeutas ocupacionales por las condiciones de vida de los sujetos está en el origen mismo de la disciplina (Mosey, 1985), no fue hasta los años 60-70 en los que en un contexto latinoamericano caracterizado "[...] por grandes debates políticos asociados a proyectos de transformación social y por la disputa de modelos de desarrollo a impulsar en todos los países de la región" (Guajardo Córdoba, 2017) se inician una serie de prácticas para atender las necesidades ocupacionales de las personas que viven situaciones de mayor vulnerabilidad social (Lopes et al., 2015; Algado et al., 2016); ha sido a partir de las dos últimas décadas cuando se han ido expandiendo en otros territorios como Estados Unidos, Canadá o Europa el interés de los terapeutas ocupacionales por la descripción y el análisis de las ocupaciones que tienen lugar en contextos de mayor vulnerabilidad y precariedad social en los que el desempeño y la participación ocupacional en condiciones de salud, bienestar y sentido personal pueden verse comprometidos de forma significativa (Gerlach, 2015; Guajardo \& Mondaca, 2017; Salas et al., 2015; Stadnyk et al., 2010). En este sentido, en los última década se han ampliado los abordajes de intervención que superen los planteamientos más reduccionistas de la disciplina que ponen el foco sólo en el individuo para pasar a una mirada que apueste por las prácticas comunitarias para la mejora y transformación de sus condiciones de vida cotidiana apostando por prácticas con un carácter más grupal y colectivo (Creek, 2017; Creek \& Cook, 2017; Vinzón et al., 2020; Zango Martín, 2017).

A la hora de atender a aquellos contextos en los que la pérdida de libertad restringe la participación y el adecuado desempeño de ocupaciones saludables y culturalmente vinculantes los esfuerzos se han centrado en primer lugar en contextos penitenciarios (Crabtree et al., 2016; Meáulle \& La Cueva, 2013) y en segundo lugar en las situaciones que experimentan poblaciones refugiadas ante diferentes situaciones políticas, sociales y/o ambientales (Blankvoort et al., 2018; Cipriani et al., 2020; Darawsheh, 2019; Trimboli \& Halliwell, 2018). En este artículo, se hará referencia a este segundo tipo de situaciones.

La convención de Ginebra sobre el Estatuto de los Refugiados de 1951 establece en su artículo 1.A.2 que una persona refugiada es aquella que

[...] debido a fundados temores de ser perseguido por motivos de raza, religión, nacionalidad, pertenencia a un determinado grupo social u opiniones 
políticas, se encuentre fuera del país de su nacionalidad y no pueda o, a causa de dichos temores, no quiera acogerse a la protección de su país; o que careciendo de nacionalidad y hallándose, a consecuencia de tales acontecimientos fuera del país donde antes tuviera su residencia habitual, no pueda o, a causa de dichos temores no quiera regresar a él.

La experiencia de desplazamiento y/o refugio causa un importante impacto sobre la vida cotidiana de los sujetos (Bennett et al., 2012; Hoare et al., 2017), ocasionando importantes modificaciones en aspectos fundamentales para los seres humanos como su identidad (Huot \& Rudman, 2010), el cambio en el desempeño de los roles como el ejercicio de la paternidad (Merry et al., 2017), su salud y su bienestar (Huot et al., 2016). El tiempo más o menos prologando de permanencia en asentamientos hace que los sujetos experimenten importantes problemas para adaptarse al nuevo contexto de vida que en ocasiones puede acompañarse de otros acontecimientos como violación, secuestro, separaciones forzosas o torturas (Boyle, 2014). De igual manera, la carencia de recursos económicos o la pérdida de actividad productiva que pueda generar suficientes ingresos de manera estable ocasiona una dependencia extensa en los recursos externos (organismos gubernamentales, ONGs, etc.) que menoscaba aspectos vitales como la causalidad personal o el locus de control interno en la vida cotidiana (McElroy et al., 2012).

Por otro lado, cabe destacar que, en contextos de crisis migratorias, de desplazamientos forzados, las personas más vulnerables ${ }^{1}$ (Feito, 2007) van a ser los grandes perjudicados. En este sentido, como destaca el Alto Comisionado de las Naciones Unidas para los Refugiados (2015, p. 18), “[...] se ha demostrado que en las operaciones sobre el terreno a menudo falta capacidad de apoyo a las personas con discapacidad en situaciones de riesgo y emergencia humanitaria”, por lo que la tarea de la Terapia Ocupacional va a resultar clave a la hora de posibilitar el desarrollo de vidas de dignidad en contextos en los que se carece de los recursos más básicos y en los que se atenta de manera constante a los derechos humanos.

\section{La Mirada a las Ocupaciones en Contextos no Occidentales}

De manera tradicional, la Terapia Ocupacional ha utilizado planteamientos y modelos occidentales a la hora de explicar y comprender las ocupaciones humanas dejando al margen aquellas conceptualizaciones y cosmovisiones que conceptualizan tanto a los seres humanos, como a sus identidades y vidas cotidianas desde otras miradas (Hammell \& Iwama, 2012).

En este sentido, la tradición de los modelos conceptuales de la terapia ocupacional persigue representaciones occidentales de la ocupación y de la salud (Kielhofner, 2009) que desde una mirada positivista y reduccionista enfatizan a un sujeto separado del contexto en el que se desempeńa, en el que parece que las actividades que lleva a cabo en su vida cotidiana no dependen de las ocupaciones que desarrollan los sujetos que le

\footnotetext{
${ }^{1}$ Vulnerabilidad asociada a las condiciones del medio (ambiental, social o de otro tipo) en que su vida se desarrolla. En este sentido, nos referimos a aquellos grupos de personas que, a consecuencia de las condiciones del medio en que viven, están en una situación de mayor susceptibilidad al daño.
} 
rodean y de un entorno que proporciona oportunidades pero, de igual manera, puede convertirse en un espacio cargado de obstáculos en ocasiones infranqueables por los sujetos que experimentan situaciones vitales más desventajosas. Sin embargo, en las últimas décadas la Terapia Ocupacional se ha visto interpelada a repensar las ocupaciones y repensarse a sí misma para posibilitar la construcción de perspectivas críticas y emancipadoras (Galheigo, 2020). Dentro de estas nuevas aportaciones cobra una especial relevancia las denominadas "Terapias Ocupacionales del Sur" (Salas et al., 2015; Algado et al., 2016) que Valderrama (2019) caracteriza como esencialmente sociales, políticas, feministas, descolonizantes en sus conocimientos y prácticas, y cuyos fundamentos éticos podrían ser los derechos humanos, siendo las ocupaciones colectivas una expresión.

De esta forma, cuando la terapia ocupacional se lleva a cabo en sociedades en las que la cultura es diferente y las realidades que viven se encuentran tan alejadas de las del mundo occidental, esas concepciones deben cambiar. Se hace necesario tener en cuenta sus propias descripciones, los recursos que tienen, tanto económicos, como materiales, sus situaciones personales y familiares. Debemos aprender a trabajar con ellos desde su mirada, responder a las necesidades que tienen realmente, sin dar por hecho que deben ser similares a las nuestras. Nosotros somos los extraños, los que estamos fuera de contexto, los extranjeros y visitantes. El profesional tiene que ser también capaz de parar y hacer un ejercicio constante de revisión de los dilemas éticos a los que se enfrenta a la hora de desarrollar una investigación cualitativa en el ámbito de la cooperación al desarrollo que pueden resultar un asunto sumamente delicado y comprometido (Pulido Fuentes, 2017). Por ello, se hace necesario conocer su cultura y tener en cuenta sus experiencias personales, los significados que las familias refugiadas narran sobre lo que les sucede, entendiendo, además como plantea Pollard (2017, p. 68) que “[...] la cultura puede ser vista como la expresión o el producto del hacer colectivo que resulta del enfoque de la comunidad en el propósito humano". De ese modo, nos aseguraremos de que nuestra presencia en el campo no supondrá una molestia y nuestra profesión puede llegar a ser relevante en su entorno. Como apuntaba Inda Zango Martín \& Pedro Moruno Miralles (Zango Martín \& Moruno Miralles, 2013, p. 10) es necesario:

[...] consolidar una actitud flexible y cómoda con el cuestionamiento del desarrollo teórico práctico de la terapia ocupacional y fomentar una identidad fluida y crítica que pueda ser significativa en los diferentes contextos en los que se ubica [...] apostando por la reflexión compartida, junto con los actores implicados, que favorezca una perspectiva intercultural de la terapia ocupacional descolonizante y descolonizada que ofrezca abordajes eficaces terapéuticamente en los contextos en los que se desarrolla.

\section{Terapia Ocupacional con Personas Desplazadas}

En los últimos años, la World Federation of Occupational Therapists (WFOT) ha prestado una especial atención a las posibilidades que la Terapia Ocupacional puede tener con personas desplazadas y/o refugiadas. Primero con la publicación de la "Declaración de Posicionamiento: Desplazamiento Humano" (World Federation of Occupational Therapists, 2014, p. 3) en la que manifiestan la necesidad de apoyar a los 
profesionales que se dedican a este ámbito para desarrollar aún más sus capacidades ya que "[...] el acceso al aporte de la terapia ocupacional es algo nuevo para la mayoría de las organizaciones que se ocupan del desplazamiento dentro de contextos tales como desastres, conflictos, campamentos de refugiados, zonas urbanas y asentamientos" y más recientemente con la publicación del "Resource Manual: Occupational Therapy for Displaced Persons" (World Federation of Occupational Therapists, 2019) en el que se proporciona conocimientos y recursos para los terapeutas ocupacionales que trabajan con poblaciones desplazadas o que están considerando la posibilidad de hacerlo.

Por otro lado, cabe destacar el trabajo desarrollado por Occupational Opportunities for Refugees and Asylum Seekers (OOFRAS), una red internacional de terapeutas ocupacionales que atienden personas desplazadas por la guerra y la persecución. Esta red buscar abordar las necesidades y derechos ocupacionales de las personas desplazadas, y construir un fuerte campo de práctica para maximizar el impacto de la terapia ocupacional en este ámbito, teniendo en cuenta que su rol puede cubrir un amplio abanico de posibilidades (World Federation of Occupational Therapists, 2019, p. 8):

Los terapeutas ocupacionales utilizan sus conocimientos básicos para trabajar con todas las personas afectadas por el impacto de enfermedad, discapacidad, condiciones físicas y/o psicológicas. Los terapeutas ocupacionales pueden proporcionar servicios directos, servicios indirectos, como el coordinador de proyectos, o servicios informales, como ser voluntarios o entrenando a voluntarios. Los terapeutas ocupacionales pueden asociarse con organismos locales para abogar y prestar servicios.

\section{Población Refugiada Saharaui}

Según el ACNUR, más de 165.000 refugiados saharauis se asientan en la actualidad en los campamentos de Auserd, Smara, Dajla, El Aaiún y Boujador, territorio conocido como hamada argelina, declarada la zona más inhóspita del planeta. Este territorio con características extremadamente áridas y aisladas está localizado a escasos kilómetros de Tinduf, una ciudad de poco más de 50.000 habitantes.

Con un conflicto iniciado en 1975, la población saharaui lleva más de 40 años residiendo en este territorio, malviviendo gracias a la ayuda exterior, cada vez más precaria y escasa. La geopolítica internacional está haciendo mover los focos de preocupación hacia otros conflictos, haciendo que la situación en esta zona del planeta se convierta en una crisis olvidada. Los esfuerzos internacionales para una solución política han sido escasos e infructuosos. La población vive esperando una resolución satisfactoria para regresar con garantías a territorio saharaui.

El contexto es sumamente inestable, con una situación medioambiental extrema que impide plantear actividades productivas que no sean de mera supervivencia, basadas fundamentalmente en una precaria agricultura y comercio informal y una población joven cada vez más escasa que presenta unas escasas perspectivas de futuro. La Agencia Española de Cooperación Internacional para el Desarrollo (2018) plantea en su diagnóstico de la situación que 
[...] las y los jóvenes, una amplia mayoría de los cuales se ha formado fuera de los campamentos o ha tenido contacto con realidades ajenas a los mismos, muestra su frustración ante las escasas oportunidades de desarrollo profesional y de participación en la toma de decisiones que los campamentos ofrecen.

Estas condiciones impactan de manera notable en la salud de la población. Tal y como apunta Médicos del Mundo (2020):

En los campamentos, las personas refugiadas saharauis se enfrentan a numerosas dificultades para cubrir sus necesidades básicas de salud: limitado acceso a una atención sanitaria adecuada por déficit de personal sanitario cualificado y de medios de diagnósticos, falta de medicamentos y un largo etcétera. Esto se traduce en elevados índices de anemia entre las mujeres, niñas y niños principalmente, diabetes, hipertensión arterial, asma, celiaquía, glaucoma, cataratas, disfunción renal, infecciones dermatológicas, y otras enfermedades.

Por otro lado, cabe destacar que la Organización Mundial de la Salud (OMS) establece que, "[...] más de 1.000 millones de personas en el mundo, cerca del 15\% de la población mundial, viven con algún tipo de discapacidad". Por otro lado, entre 2,3 y 3,3 millones de personas desplazadas tienen algún tipo de diversidad funcional. La atención a las personas con diversidad funcional en un contexto como el de los campamentos de refugiados saharauis puede sostenerse no sólo desde los principios fundamentales de la Terapia Ocupacional sino que también se puede apoyar en la propia Convención sobre los Derechos de las Personas con Discapacidad que en su artículo 11 establece que:

Los Estados Partes adoptarán, en virtud de las responsabilidades que les corresponden con arreglo al derecho internacional, y en concreto el derecho internacional humanitario y el derecho internacional de los derechos humanos, todas las medidas necesarias para garantizar la seguridad y la protección de las personas con discapacidad en situaciones de riesgo, incluidas situaciones de conflicto armado, emergencias humanitarias y desastres naturales.

En el caso de los campamentos saharauis cabe destacar como el cuidado de las personas con diversidad funcional, muchos de ellos mutilados de guerra, recae sobre las mujeres. Por otro lado, existe una visión fatalista de las posibilidades de mejora y recuperación dados los escasos recursos de rehabilitación y apoyo que existen en el contexto.

El presente trabajo tiene como objetivo principal describir y explicar la ocupación desde un enfoque cultural en familias con hijos en situación de discapacidad que residen en un contexto privado de libertad como son los campamentos de refugiados saharauis. 


\section{Método}

Estudio descriptivo transversal, de naturaleza etnográfica (Hernández Sampieri et al., 2014), realizado durante los meses de noviembre y diciembre de 2019 en los campamentos de refugiados saharauis de Tindouf (Argelia). La etnografía permite utilizar herramientas de campo que posibilitan dialogar de una manera más directa y situada con los planteamientos fundamentales de la Terapia Ocupacional crítica y sociocomunitaria (Algado et al., 2016), centrándonos en la perspectiva de la diversidad cultural (Pastore \& Sato, 2018). En el contexto que nos ocupa, la etnografía cuestiona la descentralización del conocimiento técnico, permitiendo la escucha sensible del otro y aceptando nuevas posibilidades para diferentes formas de vida (Zango Martín \& Moruno Millares, 2013).

En el trabajo de campo, imperó la escucha y observación de lo que sucedía a nuestro alrededor, modificando de manera considerable el origen del proyecto y centrándonos en las necesidades de las familias.

\section{Participantes}

Familias que habitan en cuatro wilayas (El Aaiún, Auserd, Boujadour y Dajla) de los campamentos de refugiados saharauis de Tindouf. Todas las familias incluidas en el trabajo de campo tienen un hijo o hija en situación de discapacidad ${ }^{2}$ que participa o ha participado en el programa de Vacaciones en Paz con la Asociación de Amigos del Pueblo Saharaui de Talavera de la Reina (España). Además, son alumnos y alumnas de un colegio o centro de educación especial.

Los escenarios fueron identificados en base a las actividades programadas durante los días en los que se desarrolló el proyecto de cooperación en los campamentos. Por ello, durante el trabajo de campo se entrevistó a una decena de familias saharauis, cuya conexión partía de la presencia de un niño o una niña menor de 16 años ${ }^{3}$ con discapacidad física y/o intelectual. La interacción y observación participante con los distintos miembros de la familia y su entorno, fue posible por la convivencia que las responsables del trabajo mantuvieron durante días en hogares y centros de atención a la infancia y adolescencia.

\section{Procedimiento}

Este trabajo de campo es el resultado de un Proyecto de Cooperación al Desarrollo, financiado por la Universidad de Castilla La Mancha y cuyos socios, son los integrantes de la Asociación de Talavera de la Reina. Los datos empíricos se han recogido mediante la observación participante, entrevistas no estructuradas, notas de campo, fotografías y grabaciones en vídeo (Velázquez, 2016; Denzin \& Lincoln, 2012; Phillippi \& Lauderdale, 2018). Las responsables del trabajo en los campamentos elaboraron un

\footnotetext{
${ }^{2}$ En los campamentos de refugiados saharauis, las personas con discapacidad no son un grupo homogéneo. Tienen diferentes capacidades y necesidades, y contribuyen de diversas formas a su comunidad. Sin embargo, son más vulnerables a la discriminación, explotación y violencia. Se enfrentan a numerosas barreras (físicas, sociales y emocionales) para tener acceso a los entornos compartidos en la comunidad.

${ }^{3}$ Menor de 16 ańos. Dependiente de la autorización de los progenitores para cualquier acción, movimiento y/o actividad externa al campamento.
} 
diario de campo (Scheinvar \& Nascimento, 2017), que recogía toda la información diaria, los espacios visitados y de trabajo, las personas implicadas, el tipo de acciones y su participación en el contexto. Además, dicha información se complementaba con sentimientos y emociones experimentadas.

Estos procedimientos de investigación se realizaron de acuerdo con los principios bioéticos establecidos en el Informe Belmont, junto con la Declaración de Helsinki y el Convenio sobre los Derechos Humanos y la Biomedicina del Consejo de Europa. Todos los participantes dieron su consentimiento informado. La confidencialidad se mantuvo durante todo el estudio y sólo el equipo de investigación tuvo acceso a los datos obtenidos.

\section{Análisis}

Las entrevistas, permitieron la narración personal y en primera persona de las experiencias con relación al desarrollo de las ocupaciones (en concreto, actividades de la vida diaria), en las diferentes wilayas de los campamentos de refugiados saharauis.

Fueron grabadas en audio, transcritas en su totalidad y sometidas a procesos de comparación constante (Denzin \& Lincoln, 2012). Estos relatos se complementaron con materiales empíricos procedentes de la observación participante realizada en las casas y/o jaimas de los informantes, en espacios públicos de participación social como zocos y escuelas y en los dispensarios médicos. También, se analizaron los datos de los diarios de campo elaborados por las responsables del trabajo. La combinación de todos los datos obtenidos permitió generar significados descriptivos sobre la construcción de la ocupación en contextos privados de libertad.

El proceso de análisis e interpretación se enfoca desde posiciones fenomenológicas (Suddick et al., 2020; Zahavi, 2021), a partir de las prácticas culturales de los informantes con relación a su ocupación y los contextos que la dificultan y/o facilitan. De esta manera, se ha considerado la ocupación asumiendo una perspectiva desde la que se subrayaba la influencia de aspectos sociales y culturales en la salud a través de la ocupación, aspectos que se han agrupado bajo el concepto de justicia ocupacional, que han ido perfilándose en diferentes términos: apartheid ocupacional (Kronenberg et al., 2006), privación ocupacional (Whiteford, 2000; Whiteford et al., 2017), marginación ocupacional (Townsend \& Wilcock, 2004), desequilibrio ocupacional (Wilcock et al., 1997), alienación ocupacional (Townsend \& Wilcock, 2004). Como señala Durocher et al. (2019, p. 6): “[...] los teóricos de la justicia ocupacional argumentan que tanto la prohibición de realizar ocupaciones significativas como la obligación de realizar otras que no son significativas o que no se eligen de manera voluntaria pueden afectar el bienestar individual".

\section{Resultados}

El significado de la cultura parecía ser una parte integral de la práctica profesional (Pollard, 2017), así como las circunstancias vitales con las que convivían las familias con miembros en situación de discapacidad. La combinación de ambas obligó a las responsables del proyecto a avanzar hacia la descolonización de la profesión de la Terapia 
Ocupacional, alejándose de los objetivos de intervención y centrándose en la observación para poder crear espacios de intercambio de conocimientos.

[...] un gran patio de arena, rodeado de habitaciones con alfombra y una mesa. Nińos y niñas se sentaron en el suelo alrededor de la mesa, acompañados por una persona adulta que sacó fichas de números y letras españolas y entregó unos botes con lapiceros de colores. Empezaron los gritos, la inquietud y el miedo de otros. Una niña se alejó y escondió su cabeza entre sus rodillas. La niña morena de pelo rizado intentaba pintar, con los ojos pegados al papel, mientras el nińo la empujaba y la persona adulta limpiaba sus mocos y la secreción de sus labios. Se escucharon palmas, los niños y las niñas corrieron al patio de arena y los adultos se unieron en una habitación, alrededor de la tetera y de la bandeja con vasos de té. Comenzaron las risas y una larga conversación en hasaní ${ }^{4}$ (Figura 1).

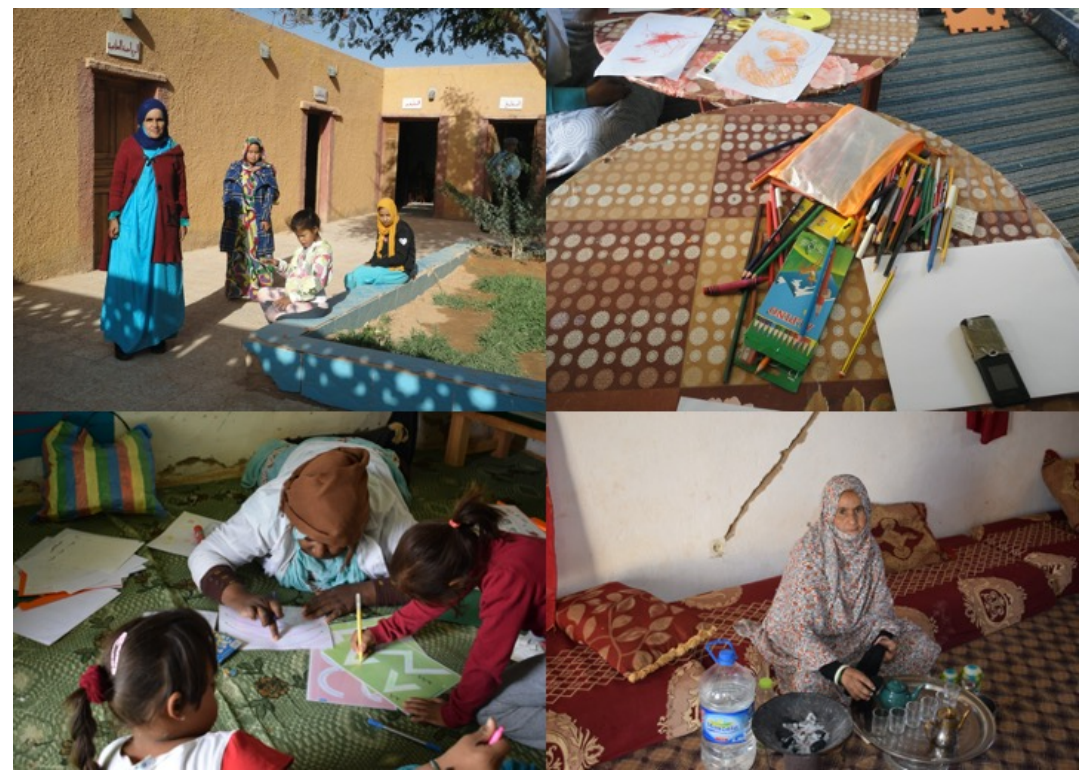

Figura 1. La cultura como parte integral de la práctica profesional.

\section{Contexto}

Los campamentos de refugiados saharauis se asientan en un territorio sumamente agreste para cuyo sostenimiento de la vida cotidiana es necesario contar con las ayudas y la financiación externa que facilitan sobrevivir a las condiciones extremas del asentamiento (Polo, 2012). Por ello, los equipos de cooperación internacional al desarrollo residen durante gran parte del año en la zona, provocando indirectamente cambios importantes en la vida diaria de la población saharaui. Estos cambios se

\footnotetext{
${ }^{4}$ Es importante destacar que, en todos los grupos de intervención de los colegios de educación especial, la directora y algunos profesionales hablan español. La mayoría de ellos, se han formado en Cuba y han vivido durante años en este país. En el caso de las familias, parte de sus integrantes han viajado a Espańa con el Programa 'Vacaciones en Paz' y/o estudian en el colegio el español como segunda lengua obligatoria. Por ello, las traducciones y la participación de las investigadoras en conversaciones no supuso ninguna limitación en ninguno de los entornos y ámbitos analizados.
} 
traducen en la modificación de sus horarios habituales para despertar o comer, en la obligatoriedad de participación a sesiones formativas, en la asistencia a controles médicos y/o analíticos o en la observación constante de hogares, colegios o centros de atención médica, entre otros.

[...] bendita la ayuda que viene. Llegan, están y se van. Te dicen qué hacer y cómo, pero se van. Aqui nos quedamos con nuestros hijos enfermos, sin medicina, sin saber, en casa, sufriendo. Esto no es para ellos (Madre).

Los campamentos se han convertido en "espacios de espera", "espacios anómalos" o “agujeros espaciotemporales” que se prolongan ya durante más de 40 años, en los que la población se ha visto abocada a normalizar su desplazamiento y asentamiento (Niebla \& Sánchez, 2018). La espera está muy presente en la vida diaria de los refugiados, observándose en forma de desidia, especialmente en las personas que habitan en la zona desde los inicios del establecimiento en los campamentos.

[...] de qué vamos a vivir. Aqui no hay nada. Esperar a vosotros, que lleguéis. No hay dinero, no se puede comprar. Estudian y no hay trabajo. No hay libertad. $Y$ ellos, qué van a hacer ellos. No los miran bien. Aqui los discapacitados no. Esperar y esperar (Directora de un colegio de educación especial).

La economía de los campamentos se construye en torno a tres pilares fundamentales: (1) recepción $\mathrm{y}$ asentamiento de cooperantes y organizaciones nacionales $\mathrm{e}$ internacionales; (2) visita y envío de dinero, material y alimentos de primera necesidad por parte de las familias de acogida, mayoritariamente españolas y (3) mercadería de gasolina, materiales de construcción y alimentos de primera necesidad.

La presencia y labor de la mujer en los campamentos es de vital importancia para el desarrollo de estos. Ellas son las responsables del mantenimiento de los hogares, de la crianza de los hijos, del cuidado de las personas mayores y de la alimentación y engorde de los animales de compañía, en su mayoría cabras. Además, lideran la preservación de la cultura saharaui ${ }^{5}$ mediante la protección y transmisión de generación en generación (Polo, 2012). Por otro lado,

[...] las mujeres saharauis, por su trayectoria en este largo conflicto, están desempeńando un importante papel en el mismo, y en el que están poniendo en valor su capacidad de compromiso, creatividad y resistencia en el proyecto político por la liberación y la independencia del pueblo saharaui (Gascón \& Ruíz Seisdedos, 2016).

[...] esto es para las madres. Nació y me abandonó. No interesa tener un hijo mal. No bien visto por el hombre. No sirve. Y qué hacer, sufrir y sufrir. Yo no puedo

\footnotetext{
${ }^{5}$ Aprovechan la toma del té y la caída del sol para sentarse junto a sus hijas. Les enseñan a coser y a bordar los telares. Las mujeres saharauis están fuertemente unidas a las telas, elemento con el que cosían y construían sus haimas y sus melfashs (vestido tradicional de la mujer saharaui). Desde los inicios de la adolescencia, les inculcan las tareas del hogar, la prestación del servicio al otro, especialmente a su marido, y la creación de recetas tradicionales. En el contexto en el que habitan, han podido conservar los tiempos de rezo, su vestimenta y la reunión de mujeres en torno a la mesa del té. Sin embargo, las haimas han sido sustituidas por casas de adobe o ladrillo cementado en la mayor parte del campamento.
} 
dormir bien. Siempre pensando en ella. Si respira, si se mueve, si tiene frío o calor. Yo no salir a ningún sitio. Yo con ella (Madre).

En la mayoría de las wilayas no existe agua corriente. El agua se suministra en camiones cisterna y es depositada en contenedores metálicos y/o de plástico, pertenecientes a diferentes grupos de familias (en su mayoría, cinco). La mayor parte de los alimentos entran a los campamentos a través de organizaciones internacionales, en forma de sacos que depositan en el punto de recogida de alimentos y al que tienen acceso las familias. En su mayoría, son alimentos de primera necesidad (zanahorias, patatas, cebollas, etc.). El contenido de cada saco se distribuye igualmente entre el grupo de familias. Las organizaciones nacionales son las encargadas de la distribución de alimentos no perecederos (pasta, arroz, azúcar, harina, etc.). Para las familias, los alimentos perecederos son de difícil acceso. Solo algunas de ellas pueden permitirse comprar carne, fruta o lácteos. El pescado es inexistente en los campamentos.

[...] esa comida no le iba bien. No engordaba. No crecía su cuerpo. Su mente no. No habia nada. El médico decía una parte de su cabeza muerta, no funciona. Vomitaba todo, la tripa, los nervios. Yo dolor cabeza, todo el día dolor cabeza. No saber qué hacer. Sola con 6 hijos. Ella no movía, ella no sentaba bien eso que llegaba (Madre).

La conexión al tendido eléctrico tampoco es viable en todas las wilayas. En aquellas que tienen acceso, se caracteriza por una red de cableado situada sobre la arena del desierto, que ha permitido que los saharauis tengan acceso a internet.

[...] ella no salir sola. Cables por suelo, se mojan y niños mueren. Ella no saber peligro. Ella anda y anda y se pierde. Yo siempre con ella. Ella llora y llora y se enfada. Ella quiere sola. No sola, peligro (Madre) (Figura 2).

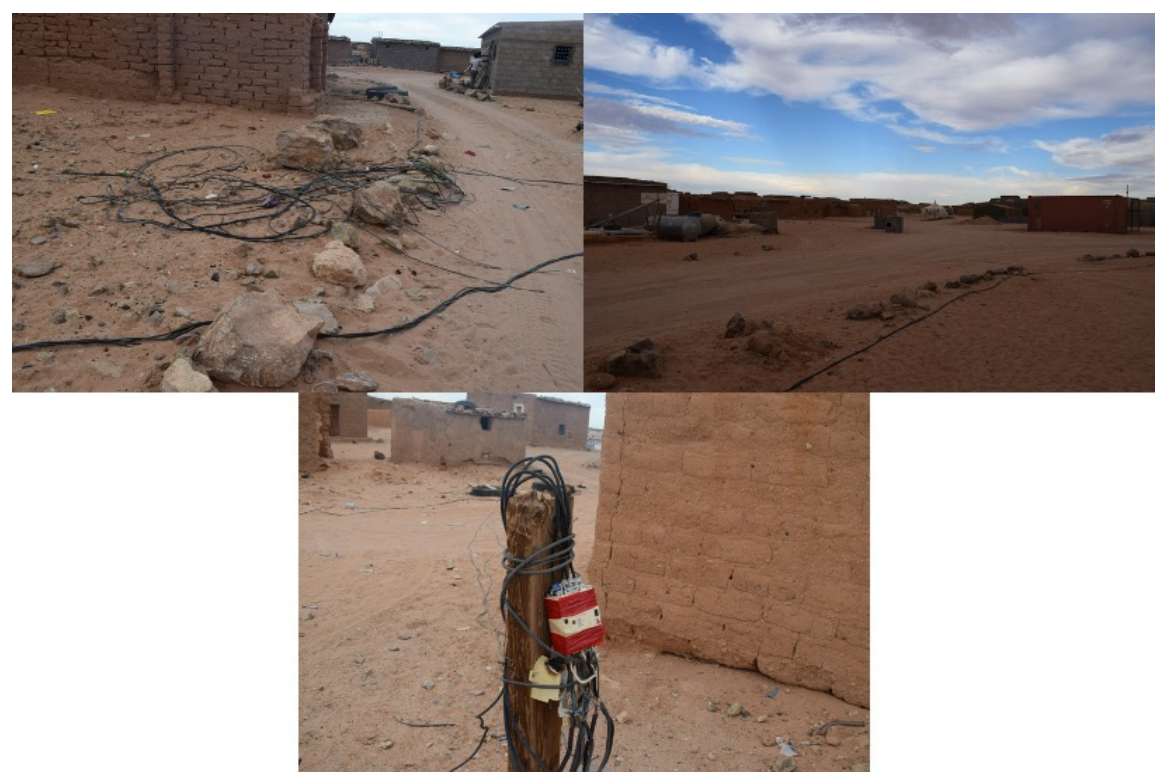

Figura 2. Contexto. 


\section{Las actividades de la vida diaria en los campamentos saharauis}

Zango Martín \& Moruno Millares (2013), argumentan que las ocupaciones significativas pueden contemplarse solo si las necesidades básicas están cubiertas. La ocupación y los procesos ocupacionales, ejes vertebrales de la Terapia Ocupacional, varían en función del contexto. El entorno, el sol y la temperatura en el desierto más inhóspito del planeta modifican sustancialmente la realización de las actividades de la vida diaria de los refugiados saharauis, así como el abastecimiento de agua, la conexión a la red eléctrica o la llegada de alimentos de primera necesidad. Por tanto, las condiciones del entorno suponen un desafío constante a la ocupación y/o la actividad.

En los campamentos, el tiempo invertido en el desarrollo de las actividades de la vida diaria se organiza a partir de una noción de "tiempo" marcado por tres tipos de actividades: (1) la luz del sol, (2) el rezo y (3) la hora del té, siendo las dos últimas las actividades más significativas de la población refugiada.

[...] observamos como los niños y niñas con discapacidad no participaban en los rezos. Todos los integrantes de la familia se alejaban de la habitación común con la llamada, pero ellos se quedan ahi sentados y/o jugando (Investigadoras).

[...] cansada, dolor de cabeza. Llega esto, el té, y todos los males se pasan. El té medicina para los saharauis. Yo mucha preocupación por ella. Ella no, aqui no. Esto no es para ella (Madre).

Whiteford (2000) afirma que las personas que habitan en campos de refugiados sufren privación ocupacional, entendiendo por esto, la falta de participación en actividades y la desconexión de una rutina diaria a consecuencia de las experiencias vividas, generando conductas desadaptativas. Siguiendo esta afirmación, cabría preguntarse si es el etnocentrismo cultural del que coopera o visita los campamentos el que impera para la adjudicación de etiquetas de índole profesional.

Observamos como los niños y las niñas con discapacidad no participaban en las actividades de la vida diaria que se realizaban en los hogares. Por la mañana, se levantaban, tomaban pan y té (a veces, un cartón pequeño de zumo), se vestían, cogían su mochila y esperaban a que llegara el coche del colegio.

Ella quiere barrer. Ella no. No lo hace bien. Ella quiere coger bandeja. Ella no. Ella quiere hacer té. Ella no. Ella peligro (Madre).

Cuando regresaban, se sentaban y jugaban con una piedra, arena o dibujaban con lapiceros de colores. Pasaban horas sentados y/o tumbados sobre la alfombra del suelo.

En las entrevistas que mantuvimos con madres y hermanas de niños y niñas con discapacidad, proyectamos la participación en las actividades significativas como símbolo de pertenencia. Transmitimos el sentimiento de pertenencia cuando ellos y ellas son parte activa del funcionamiento del núcleo familiar.

[...] pequeñas tareas pueden ayudar a que ella se calme. Puede ayudar a limpiar los platos con agua o a recoger la mesa y la bandeja. No es tan importante el resultado, sino el sentimiento de que ella pertenece a la familia y es útil. Ella 
os quiere imitar y se irrita cuando no le permitís participar en lo que para vosotros (y también para ella) es importante (Investigadoras) (Figura 3).

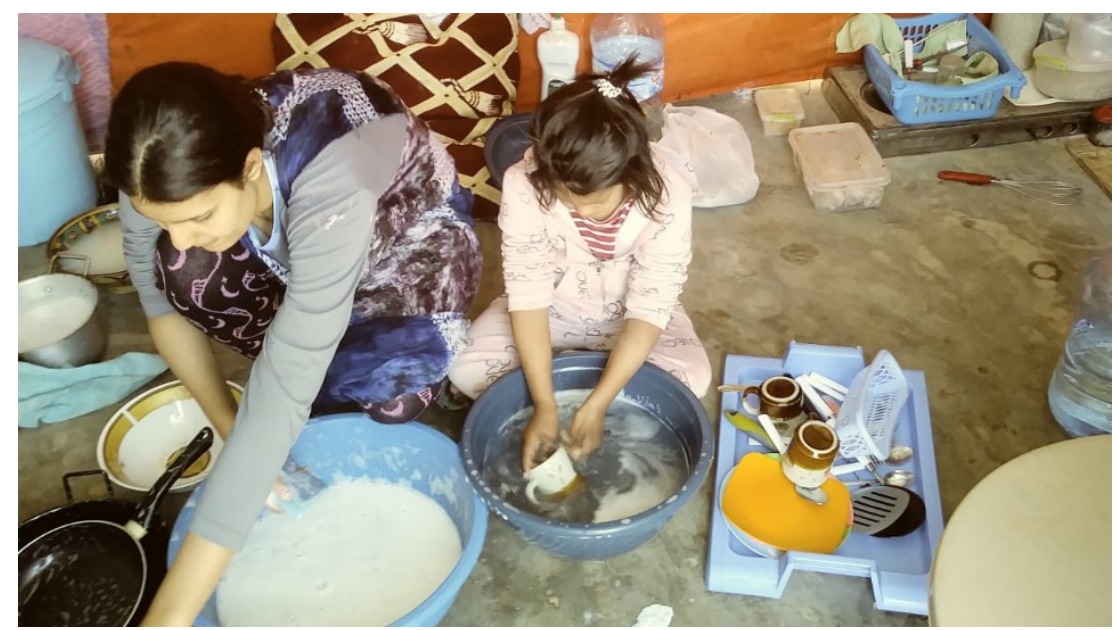

Figura 3. Participación en las actividades de la vida diaria del núcleo familiar.

\section{Presencia de agua en las actividades de la vida diaria}

La primera llamada al rezo y el amanecer marcan el inicio de la vida diaria en los campamentos de refugiados saharauis. Las mujeres parten con zuecos y melfa (vestimenta tradicional saharaui), acompañadas de sus garrafas, hacia los depósitos de agua. La cantidad de agua que corresponde a cada familia y día debe administrarse para la higiene personal, el lavado de ropa y la cocina de alimentos para todos los miembros de la casa. Por tanto, es probable que, en muchas ocasiones, la preparación y el cocinado de alimentos se anteponga a tareas de higiene personal y bucodental (Figura 4).

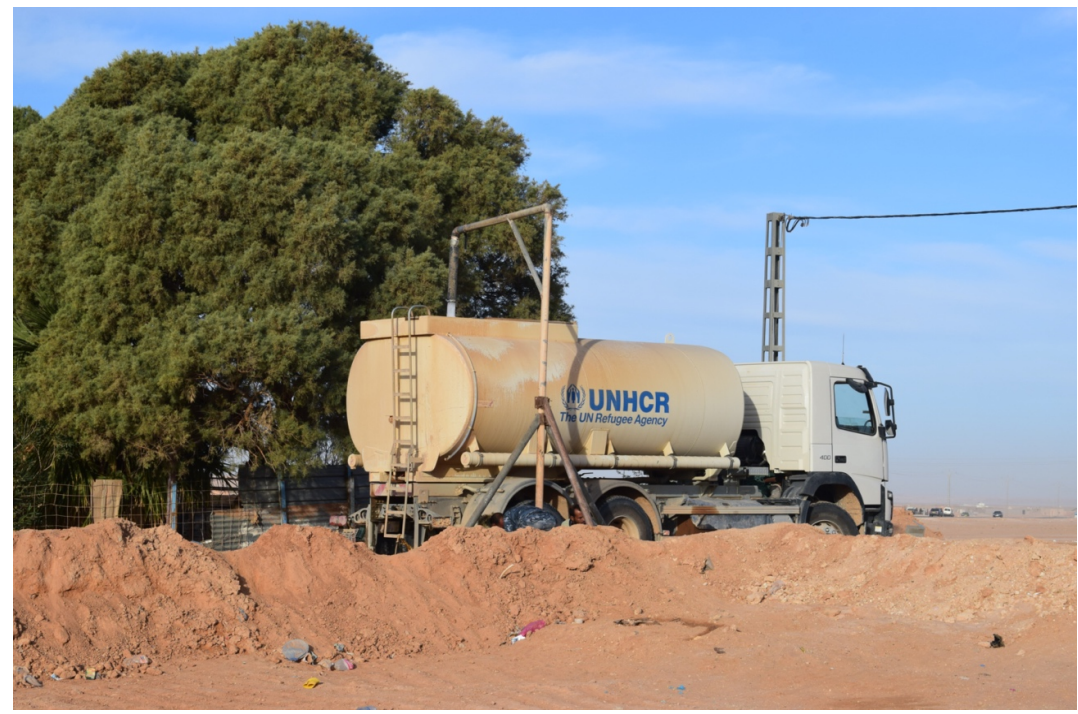

Figura 4. Presencia y administración de agua en los campamentos saharauis. 

(Madre).

Es importante destacar que todos los niños participantes en el proyecto viajan a Espańa durante los meses de verano. En algunos casos, tienden a replicar las costumbres adquiridas y/o practicadas en las familias acogedoras españolas.

[...] cuando llega es un problema. Llora y llora. Quiere salir a la calle, quiere comprar, quiere tener dinero, quiere bañar, quiere jugar como en España. Aqui no. Aqui no puede. Aqui peligro de coches, de personas que hacen mal, de cables. Aqui no dinero para comprar. Aqui no juguetes. Aqui no agua (Madre).

\section{Red eléctrica y temperatura ambiental en las actividades de la vida diaria}

La precaria instalación eléctrica y las altas temperaturas ambientales en los campamentos marcan la distribución de las tareas y actividades de la vida diaria. El tendido eléctrico deja de funcionar a media mañana y no regresa hasta la caída del sol. Las mujeres aprovechan el amanecer para retirar la arena que ha invadido todos los rincones del hogar durante la noche, para adquirir los alimentos más básicos y necesarios en el mercado, para cocinar sus platos más típicos o para tomarse el primer té del día con tranquilidad. En algunas ocasiones, los jóvenes varones, visualizan los informativos en la televisión, convirtiéndose este momento en el único contacto oficial con el exterior. Hasta el atardecer, la actividad de la mayor parte de las familias saharauis se reduce a la contemplación y conversación en el interior de sus hogares.

[...] se pone nerviosa. Aqui no luz, no tele. Ella quiere ver dibujos. Ella tele siempre. Ella calor, no salir calle. Ella no tranquila. Ella llora. A mi duele cabeza mucho. Ella peligro. Niños se rien. Echan arena en cabeza. Se rien. Yo canso. Yo dolor huesos. Yo mayor. Ella mucha actividad. Ella todo el tiempo movimiento. Aqui no. Aqui no puede. Eso en España (Madre).

\section{Diferencias sociales y económicas que repercuten en la ejecución de las actividades de la vida diaria}

La ayuda internacional es homogénea para todos los habitantes de los campamentos de refugiados. Sin embargo, algunos miembros de las familias, mayoritariamente niños de 8 a 12 años viajan anualmente a países europeos (principalmente, España e Italia) para ser acogidos en el Programa de Vacaciones en Paz. Esto conlleva que la población infantil acogida regresa a los campamentos cargando alimentos de difícil acceso en los mercados locales, tecnología de última generación y dinero, entre otros enseres. Durante el resto del año, parte de las familias europeas acogedoras dotan al hogar de utensilios de cocina que facilitan la vida diaria de las mujeres, materiales de construcción para mejorar la habitabilidad de las familias o alimentos para aumentar los índices nutricionales de los pequeńos y mayores. Esta ayuda externa e individual, financiada por cada familia acogedora acrecienta, en muchas ocasiones, las diferencias sociales y económicas entre las familias de refugiados saharauis, a la vez que permite oportunidades para el desarrollo 
del comercio local, impulsando el trabajo en jóvenes y adultos (mayoritariamente, varones) y una fuente constante de ingreso en los campamentos (Santos, 2016).

Son pocos los niños y niñas con dificultades físicas y/o intelectuales, que viajan en los meses estivales a países europeos. Esto hace que las personas con discapacidad y sus familias reciban menos recursos externos por parte de familias acogedoras, aunque se tiende a que estén más protegidos por las organizaciones nacionales e internacionales en terreno. Parte de los niños y nińas con discapacidad necesitan productos de apoyo, de alto coste y difícil acceso, así como alimentos especiales para intolerancias o atención médica especializada para dispensación de medicación o programación de intervenciones.

[...] él no mueve nada. Él sonrie cuando me ve. Él muy delgado. Él no crece. Yo digo llevar a España, pero es muy pequeño. Me dicen que no soporta avión. Me dicen que muy complicado encontrar familia de acogida. Aqui llevo a una curandera tradicional para sanar su tripa y que pueda beber sin atragantar. Garganta no. No pasa bebida y alimento. Él tose mucho. El puede morir. Aqui no silla de ruedas. Aqui cojines. Yo saco a curandera. Su madre no puede por peso. Su madre mal de parto. Yo en brazos. Sus piernas no rectas. Él no camina. Él no habla. Él mucha baba. Todo el tiempo baba. Aqui no hay médico, no fisioterapia. Aqui nada. Yo digo sacar, pero no sacar a España. Aqui se nos muere. Yo desesperado (Padre).

\section{Acceso a los servicios de salud}

La mayor parte de la población femenina y de edad avanzada, utiliza remedios tradicionales para curar y/o disminuir sus dolencias, en su mayoría provocadas por las largas jornadas sobre el suelo y por el déficit de vitaminas y alimentos de mayor carga nutricional. La medicación farmacéutica es de difícil acceso y en muchas ocasiones, requiere de un coste que los refugiados no pueden asumir. A ello, hay que añadir que los dispensarios médicos en ocasiones están alejados de los hogares o precisan de la presencia de un transporte a motor.

En las familias con nińos y niñas con discapacidad, el acceso a medicina especializada es prácticamente imposible. Dependen de las comisiones médicas internacionales, que en ocasiones no pueden satisfacer al completo la demanda por falta de medios materiales y/o tecnológicos (Figura 5).

Desde los colegios de educación especial, comunican las necesidades a través de las asociaciones nacionales e internacionales, pero en muchas ocasiones distan del problema real que presentan los nińos y las nińas con discapacidad.

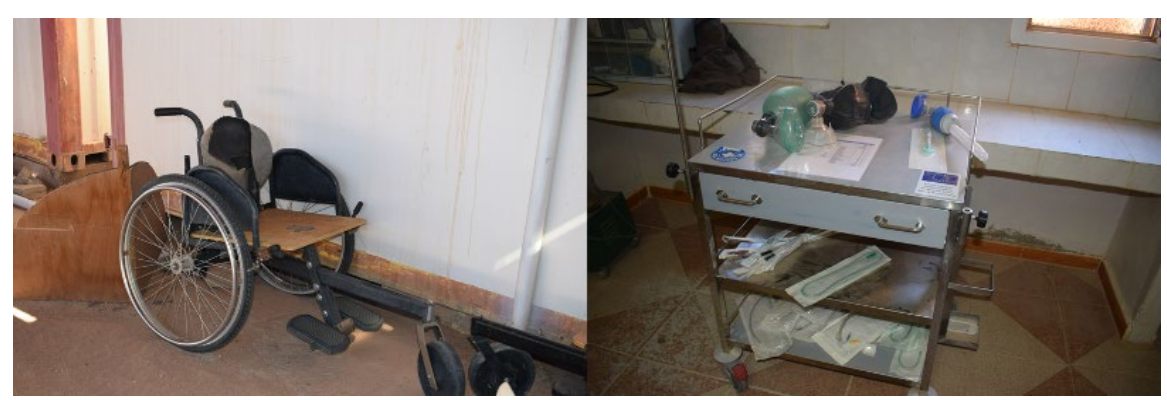

Figura 5. Acceso a servicios de salud. 
[...] es muy dificil, se puede hacer muy poco. Si a una niña se le rompen sus gafas, esa niña no ve. Hasta que regresa a España o viene la comisión médica o la asociación responsable, esa niña no ve. La familia no tiene dinero. Aqui no hay recursos. Hay que esperar, a veces, al verano y esa niña no ve. A veces, hay que operar y no es posible. No entra en lista o su operación muy dificil porque requiere cuidados después. La familia no sabe. Aqui en los colegios, no hay profesionales. Los voluntarios ayudan y casi no cobran. Muy poco dinero al año. Aqui todo muy difícil. Yo desespero. Yo no duermo. Yo no sé qué hacer. Pero la niña no ve y es un problema. La familia no ayuda. Muy difícil con las familias. Los niños con discapacidad aqui victimas. Muy difícil (Directora de colegio de educación especial).

\section{Limitaciones del estudio}

El acceso a los datos y a la información en un trabajo de estas características está sujeto al tiempo en el que las investigadoras pasan haciendo el trabajo de campo, conviviendo, observando, hablando con las familias. Ese tiempo es finito y permite acceder a una cantidad limitada de experiencias. Un mayor tiempo de convivencia posibilitaría trazar otros arcos temporales que permitan contemplar las vivencias cotidianas con mayor amplitud y profundidad estableciendo relaciones de confianza más seguras y comprometidas con la población saharaui. Además, cabe destacar que este artículo ha centrado su investigación y discurso en familias con hijos en situación de discapacidad, cuya peculiaridad dista del funcionamiento de otros miembros de la comunidad.

\section{Propuestas de futuro}

Por un lado, cabe señalar la necesidad de abordar en mayor profundidad las experiencias cotidianas de los niños y niñas con discapacidad y sus familias en un contexto como el de los campamentos de refugiados saharauis, sus oportunidades, barreras y sus esfuerzos por construir una vida con dignidad en un espacio de suma dureza. De igual manera sería de enorme relevancia ampliar algunos de los aspectos que en nuestro trabajo de campo ha supuesto sólo una aproximación como es la participación ocupacional de las mujeres y de forma más específica de las mujeres en situación de discapacidad.

Por otro, hay que analizar si los apoyos recibidos por organizaciones internacionales son efectivos y permiten gestionar los recursos propios del terreno. Cabe destacar en este sentido, si las políticas de formación en oficios para mujeres saharauis permiten la subsistencia de las familias y de la comunidad.

\section{Conclusiones}

La vida cotidiana de las personas que residen en los campamentos de refugiados está determinada por un contexto sumamente hostil y agreste que dificulta las condiciones de salud, bienestar y dignidad. La espera mantenida durante ańos para una solución política que posibilite un futuro impacta de manera decisiva sobre las ocupaciones que se vuelven sumamente precarias y muy ligadas al apoyo externo por parte de organismos 
internacionales u ONGS, que en tiempos de pandemia global escasean y provocan en la comunidad brechas y dificultades críticas para acceder a productos de primera necesidad, educación o medicinas.

Sin embargo, en medio de este inhóspito entorno, las personas buscan posibilidades para la participación ocupacional que proporciona vías para el mantenimiento de lo colectivo, la perpetuación de la memoria y la identidad gracias a la conservación de ritos, narrativas y experiencias.

La Terapia Ocupacional debe verse interpelada a ofrecer oportunidades de ocupación más digna para todas las personas, apoyando y protegiendo los derechos de participación ocupacional de todos los sujetos, especialmente de aquellos que experimentan situaciones de mayor vulnerabilidad. Esto exige un esfuerzo de comprensión de los contextos y realidades en los que se producen las ocupacionales apostando por enfoques comunitarios que dejen atrás planteamientos más individualistas y reduccionistas.

\section{Agradecimientos}

Queremos agradecer a la Asociación del Pueblo Saharaui de Talavera y a todas las personas que residen en las Wilayas por prestarnos sus tiempos, sus palabras y sus espacios de vida cotidiana.

\section{Referencias}

Agencia Española de Cooperación Internacional para el Desarrollo - AECID. (2018). Estrategia humanitaria 2018-2019: población refugiada saharaui. Espanha: Cooperación Española.

Algado, S. S., Córdoba, A. G., García-Ruiz, S., Oliver, F. C., \& Galheigo, S. M. (2016). Terapias ocupacionales desde el sur: derechos humanos, ciudadania y participación. Santiago: Mayo.

Alto Comisionado de las Naciones Unidas para los Refugiados - ACNUR. (2015). Estudio temático sobre los derechos de las personas con discapacidad en virtud del artículo 11 de la Convención sobre los Derechos de las Personas con Discapacidad, relativo a las situaciones de riesgo y emergencias humanitarias: informe de la Oficina del Alto Comisionado de las Naciones Unidas para los Derechos Humanos. Espanha: Consejo de Derechos Humanos.

Bennett, K. M., Scornaiencki, J. M., Brzozowski, J., Denis, S., \& Magalhaes, L. (2012). Immigration and its impact on daily occupations: a scoping review. Occupational Therapy International, 19(4), 185203. http://dx.doi.org/10.1002/oti.1336.

Blankvoort, N., Arslan, M., Tonoyan, A., Damour, A. Q., \& Mpabanzi, L. (2018). A new you: a collaborative exploration of occupational therapy's role with refugees. World Federation of Occupational Therapists Bulletin, 74(2), 92-98. http://dx.doi.org/10.1080/14473828.2018.1526560.

Boyle, M. (2014). Occupational performance and self-determination: the role of the occupational therapist as volunteer in two mountain communities. Australian Occupational Therapy Journal, 61(1), 6-12. http://dx.doi.org/10.1111/1440-1630.12104.

Cipriani, J., Davis, M., Gralinski, E., Monforte, S., \& Strausser, J. (2020). Examining the occupational needs and OT intervention strategies used with refugee populations: a scoping review. The American Journal of Occupational Therapy, 74(Suppl. 1), 7411505203p1. http://dx.doi.org/10.5014/ajot.2020.74S1-PO7508.

Crabtree, J. L., Wall, J. M., \& Ohm, D. (2016). Critical reflections on participatory action research in a prison setting. OTJR, 36(4), 244-252. http://dx.doi.org/10.1177/1539449216669132.

Creek, J. (2017). Enabling occupational therapy practice in marginal settings. In D. Sakellariou \& N. Pollard (Eds.), Occupational therapies without borders: integrating justice with practice (pp. 237-244). New Jersey: Elsevier. 
Construyendo y entendiendo la ocupación en contextos privados de libertad: experiencias de familias con hijos en situación de discapacidad en campamentos de refugiados

Creek, J., \& Cook, S. (2017). Learning from the margins: enabling effective occupational therapy. British Journal of Occupational Therapy, 80(7), 423-431. http://dx.doi.org/10.1177/0308022617701490.

Darawsheh, W. B. (2019). Exploration of occupational deprivation among syrian refugees displaced in Jordan. American Occupational Therapy Association, 73(4), 7304205030p1. http://dx.doi.org/10.5014/ajot.2019.030460.

Denzin, N. K., \& Lincoln, Y. S. (2012). El campo de la investigación cualitativa: manual de investigación cualitativa I. Barcelona: Gedisa.

Durocher, E., Gibson, B. E., \& Rappolt, S. (2019). Justicia ocupacional: una revisión de conceptos. Journal of Occupational Science, 21(4), 1-13. http://dx.doi.org/10.1080/14427591.2019.1616359.

Feito, L. (2007). Vulnerabilidad. Anales del Sistema Sanitario de Navarra, 30(Suppl. 3), 7-22.

Galheigo, S. M. (2020). Terapia ocupacional, cotidiano e a tessitura da vida: aportes teórico-conceituais para a construção de perspectivas críticas e emancipatórias. Cadernos Brasileiros de Terapia Ocupacional, 28(1), 5-25. http://dx.doi.org/10.4322/2526-8910.ctoAO2590.

Gascón, M. L. G., \& Ruíz Seisdedos, S. (2016). Análisis del conflicto saharaui desde una perspectiva de género. Index de Enfermería, 25(3), 185-189.

Gerlach, A. J. (2015). Sharpening our critical edge: occupational therapy in the context of marginalized populations. Canadian Journal of Occupational Therapy, 82(4), 245-253. http://dx.doi.org/10.1177/0008417415571730.

Guajardo Córdoba, A. G. (2017). Lecturas y relatos históricos de la terapia ocupacional en Suramérica: una perspectiva de reflexión crítica. Revista Ocupación Humana, 16(2), 110-117. http://dx.doi.org/10.25214/25907816.141.

Guajardo, A., \& Mondaca, M. (2017). Human rights, occupational therapy and the centrality of social practices. In D. Sakellariou \& N. Pollard (Eds.), Occupational therapies without borders: integrating justice with practice (pp. 102-108). New Jersey: Elsevier.

Hammell, K. R. W., \& Iwama, M. K. (2012). Well-being and occupational rights: an imperative for critical occupational therapy. Scandinavian Journal of Occupational Therapy, 19(5), 385-394. http://dx.doi.org/10.3109/11038128.2011.611821.

Hernández Sampieri, R., Fernández Collado, C., \& Baptista Lucio, P. (2014). Metodología de la investigación. México: McGraw-Hill Education

Hoare, T., Vidgen, A., \& Roberts, N. (2017). In their own words: a synthesis of the qualitative research on the experiences of adults seeking asylum. A systematic review of qualitative findings in forced migration. Medicine, Conflict, and Survival, 33(4), 273-298. http://dx.doi.org/10.1080/13623699.2017.1419902.

Huot, S., \& Rudman, D. L. (2010). The performances and places of identity: conceptualizing intersections of occupation, identity and place in the process of migration. Journal of Occupational Science, 17(2), 68-77. http://dx.doi.org/10.1080/14427591.2010.9686677.

Huot, S., Kelly, E., \& Park, S. J. (2016). Occupational experiences of forced migrants: a scoping review. Australian Occupational Therapy Journal, 63(3), 186-205. http://dx.doi.org/10.1111/1440-1630.12261.

Kielhofner, G. (2009). Conceptual foundations of occupational therapy practice. Filadelfia: FA Davis.

Kronenberg, F., Salvador, S. A., \& Pollard, N. (2006). Terapia ocupacional sin fronteras: aprendiendo del espiritu de supervivientes. Madrid: Médica Panamericana.

Lopes, R. E., Malfitano, A. P. S., Silva, C. R., \& Borba, P. (2015). Historia, conceptos y propuestas en la terapia ocupacional social de Brasil. Revista Chilena de Terapia Ocupacional, 15(1), 73. http://dx.doi.org/10.5354/0719-5346.2015.37132.

McElroy, T., Muyinda, H., Atim, S., Spittal, P., \& Backman, C. (2012). War, displacement and productive occupations in northern uganda? Journal of Occupational Science, 19(3), 198-212. http://dx.doi.org/10.1080/14427591.2011.614681.

Meáulle, D. E., \& La Cueva, C. M. F. (2013). El valor de las ocupaciones en prisión: planteamientos éticos y críticos para una intervención transformadora. Revista TOG, 10(17), 1-28.

Médicos del Mundo. (2020). Médicos del Mundo en los campamentos de refugiados saharauis. Recuperado el 5 de septiembre de 2020, de https://www.medicosdelmundo.org/que-hacemos/africa/argeliacampamentos-en-tinduf 
Construyendo y entendiendo la ocupación en contextos privados de libertad: experiencias de familias con hijos en situación de discapacidad en campamentos de refugiados

Merry, L., Pelaez, S., \& Edwards, N. C. (2017). Refugees, asylum-seekers and undocumented migrants and the experience of parenthood: a synthesis of the qualitative literature. Globalization and Health, 13(1), 75. http://dx.doi.org/10.1186/s12992-017-0299-4.

Mosey, A. C. (1985). Eleanor Clarke Slagle lecture, 1985: a monistic or a pluralistic approach to professional identity? The American Journal of Occupational Therapy, 39(8), 504-509. http://dx.doi.org/10.5014/ajot.39.8.504.

Niebla, S. A., \& Sánchez, C. A. (2018). Identidades en desastres prolongados. Escenarios transnacionales desde los campamentos de refugiados Saharauis. Papeles Del CEIC - International Journal on Collective Identity Research, 2018(1), 1-28. http://dx.doi.org/10.1387/pceic.17661.

Pastore, M. D. N., \& Sato, M. T. (2018). The patterns of socio-cultural diversity: dialogues among occupational therapy, Africa and ethnography. Cadernos Brasileiros de Terapia Ocupacional, 26(4), 952-959. http://dx.doi.org/10.4322/2526-8910.ctoARF1240.

Phillippi, J., \& Lauderdale, J. (2018). A guide to field notes for qualitative research: context and conversation. Qualitative Health Research, 28(3), 381-388. http://dx.doi.org/10.1177/1049732317697102.

Pollard, N. (2017). Re!exiones sobre ocupación, identidades culturales y transformación social. Revista Ocupación Humana, 17(1), 55-72. http://dx.doi.org/10.25214/25907816.155.

Polo, C. J. (2012). Resistencias cotidianas de las refugiadas saharauis. Granada: Universidad de Granada.

Pulido Fuentes, M. (2017). Os dilemas próprios de uma pesquisa qualitativa na área da cooperação para o desenvolvimento: uma questão delicada. Acta Bioethica, 23(1), 109-117. http://dx.doi.org/10.4067/S1726569X2017000100109.

Salas, E. N., Garlito, P. C., Córdoba, A. G., Prado, R. S., \& Miralles, P. M. (2015). Terapia ocupacional y exclusión social: hacia una praxis basada en los derechos humanos. España: Editorial Segismundo.

Santos, O. A. (2016). La última wilaya la diáspora saharaui. comunidad en la frontera (Trabajo final de máster). Universidad de Barcelona, Barcelona.

Scheinvar, E., \& Nascimento, M. L. (2017). Diario de campo: el autor como protagonista. Pesquisas e Práticas Psicossociais, 12(4), 1-7.

Stadnyk, R., Townsend, E., \& Wilcock, A. (2010). Occupational justice. In E. A. Townsend \& C. H. Christiansen (Eds.), Introduction to occupation: the art and science of living (pp. 329-358). New Jersey: Pearson Education.

Suddick, K. M., Cross, V., Vuoskoski, P., Galvin, K. T., \& Stew, G. (2020). The work of hermeneutic phenomenology. International Journal of Qualitative Methods, 19, 1-14. http://dx.doi.org/10.1177/1609406920947600.

Townsend, E., \& Wilcock, A. A. (2004). Occupational justice and client-centred practice: a dialogue in progress. Canadian Journal of Occupational Therapy, 71(2), 75-87. http://dx.doi.org/10.1177/000841740407100203.

Trimboli, C., \& Halliwell, V. (2018). A survey to explore the interventions used by occupational therapists and occupational therapy students with refugees and asylum seekers. World Federation of Occupational Therapists Bulletin, 74(2), 106-113. http://dx.doi.org/10.1080/14473828.2018.1535562.

Valderrama, C. M. V. (2019). South occupational therapies: a proposal for its understanding. British Journal of Occupational Therapy, 27(3), 671-680. http://dx.doi.org/10.4322/25268910.ctoARF1859.

Velázquez, B. B. (2016). Taller de investigación cualitativa. Madrid: UNED.

Vinzón, V., Allegretti, M., \& Magalhães, L. (2020). Um panorama das práticas comunitárias da terapia ocupacional na América Latina. Cadernos Brasileiros de Terapia Ocupacional, 28(2), 600-620. http://dx.doi.org/10.4322/2526-8910.ctoAR1891.

Whiteford, G. (2000). Occupational deprivation: global challenge in the new millennium. British Journal of Occupational Therapy, 63(5), 200-204. http://dx.doi.org/10.1177/030802260006300503.

Whiteford, G., Townsend, E., Bryanton, O., Wicks, A., \& Pereira, R. (2017). The participatory occupational justice framework: salience across contexts. In D. Sakellariou \& N. Pollard (Eds.), 
Construyendo y entendiendo la ocupación en contextos privados de libertad: experiencias de familias con hijos en situación de discapacidad en campamentos de refugiados

Occupational therapies without borders: integrating justice with practice (pp. 163-174). Amsterdan: Elsevier.

Wilcock, A. A., Chelin, M., Hall, M., Hamley, N., Morrison, B., Scrivener, L., Townsend, M., \& Treen, K. (1997). The relationship between occupational balance and health: A pilot study. Occupational Therapy International, 4(1), 17-30. http://dx.doi.org/10.1002/oti.45.

World Federation of Occupational Therapists - WFOT. (2014). Declaración de posicionamiento: desplazamiento humano (revisado). Recuperado el 5 de septiembre de 2020, de https://www.wfot.org

World Federation of Occupational Therapists - WFOT. (2019). Resource manual: occupational therapy for displaced persons. London: WFOT.

Zahavi, D. (2021). Applied phenomenology: why it is safe to ignore the epoché. Continental Philosophy Review, 54(2), 259-273. http://dx.doi.org/10.1007/s11007-019-09463-y.

Zango Martín, I. (2017). Terapia ocupacional comunitaria. Madrid: Editorial Sintesis.

Zango Martín, I., \& Moruno Millares, P. (2013). Aportaciones de la etnografía doblemente reflexiva en la construcción de la terapia ocupacional desde una perspectiva intercultural. Antropólogos Iberoamericanos en Red, 8(1), 9-48. http://dx.doi.org/10.11156/aibr.080102.

\section{Contribución de los Autores}

Pablo Cantero-Garlito, Marta Rodríguez-Hernández y Carmen Cipriano-Crespo concibieron, analizaron y contribuyeron en la redacción del texto. Todos los autores aproban su versión final.

\section{Fuente de Financiamiento}

El trabajo de campo ha sido realizado gracias a la financiación del Vicerrectorado de Internacionalización y Formación Permanente de la Universidad de Castilla La Mancha [208500.422D.48000].

\section{Autor para la correspondencia}

Marta Rodríguez-Hernández

e-mail: marta.rhernandez@uclm.es

\section{Editor de sección}

\section{Profa. Dra. Carmen Aleida Fernández Moreno}

